\title{
How Is Helicity (and Twist) Partitioned in Magnetohydrodynamic Simulations of Reconnecting Magnetic Flux Tubes?
}

\author{
James Threlfall (1), Andrew N. Wright (iD, and Alan W. Hood (1) \\ School of Mathematics and Statistics, Mathematical Institute, University of St Andrews, St Andrews, KY169SS, UK; jwt9@st-andrews.ac.uk \\ Received 2020 May 14; revised 2020 June 9; accepted 2020 June 11; published 2020 July 16
}

\begin{abstract}
Magnetic helicity conservation provides a convenient way to analyze specific properties (namely, the linkage and twist) of reconnecting flux tubes and yield additional insight into the pre- and post-reconnection states of magnetic structures in the solar atmosphere. A previous study considered two flux tubes with footpoints anchored in two parallel planes. They showed that reconnection would add self-helicity equivalent to a half turn of twist to each flux tube. We address a related and fundamental question here: if two flux tubes anchored in a single plane reconnect, what are the resulting twists imparted to each of the reconnected tubes? Are they equal and do they have a simple exact value independent of footpoint location? To do this, we employ a new (computationally efficient) method which subdivides each flux tube into distinct elements and calculates the mutual helicity of many elemental pairs, the sum of which determines the self-helicity of the overall flux tube. Having tested the method using a simple analytical model, we apply the technique to a magnetohydrodynamic simulation where initially untwisted magnetic flux tubes are sheared and allowed to reconnect (based on a previous reconnection model). We recover values of self-helicity and twist in the final end state of the simulations which show excellent agreement with theoretical predictions.
\end{abstract}

Unified Astronomy Thesaurus concepts: Magnetic fields (994); Solar magnetic reconnection (1504); Magnetohydrodynamical simulations (1966); Magnetohydrodynamics (1964)

\section{Introduction}

Magnetic helicity is a powerful mathematical tool to analyze the magnetic topology of specific structures in many different physical systems. For example, the fact that helicity is approximately conserved during magnetic reconnection events has led to its wide use in plasma physics (Taylor 1974), including terrestrial magnetospheric (Wright \& Berger 1989) or solar coronal phenomena (Hood et al. 2009; Priest et al. 2016). The calculation of magnetic helicity itself also depends on the specific application: the classic concept of a volume-integrated quantity (Moffatt 1969) has since been adapted to analyze the helicity of constituent flux elements (e.g., flux tubes; Berger \& Field 1984) or indeed individual field lines (Berger 1988; Russell et al. 2015; Yeates \& Hornig 2016). Developments like relative helicity (Berger \& Field 1984) have led to elegant or relatable properties and interpretations, which also allow further insight into the evolution of specific physical systems, in our case, solar flares.

One of the key unanswered questions in solar physics surrounds the formation of highly twisted flux ropes which ultimately erupt as a solar flare and/or coronal mass ejection (CME). The amount of twist seen in the preeruptive state is, in many cases, often much smaller than that seen following the propagation of the structure as an interplanetary CME (Webb 2000; Démoulin 2008; Vourlidas 2014). This discrepancy between pre- and post-reconnection twist naturally suggests magnetic helicity as a likely route to provide further insight. Magnetic helicity conservation introduces an additional constraint in the eruption process, as the slow buildup of helicity prior to an eruption must also be reflected in the energy and twist partitioning in the final post-eruption state (both in the ejected and tethered features seen). Post-flare eruption configurations do not appear to relax to a potential state, as one might expect (given the large amount of energy released from the field during a flare); the presence of sheared post-flare loops (e.g., Asai et al. 2004; Warren et al. 2011; Aulanier et al. 2012) hint at some additional property playing a key role in the redistribution process.

The application of magnetic helicity conservation as a way to further constrain theoretical magnetic reconnection models in the context of eruptive flares remains in relative infancy (while also becoming a key aspect in understanding the large-scale or global coronal evolution; e.g., Démoulin et al. 2002; Mackay \& van Ballegooijen 2006; Yeates \& Mackay 2009; Mackay et al. 2011). A common assumption among recent applications to eruptive flares is helicity equipartition; reconnection, and the associated release of mutual helicity, is assumed to contribute equally to the self-helicity of the reconnected flux tubes (e.g., Priest et al. 2016; Priest \& Longcope 2017). This assumption has proven to be valid in the context of magnetic reconnection of flux tubes which extend between two parallel planes (Wright \& Berger 1989), but in a more solar-relevant framework (where reconnecting tubes are anchored in the same plane), this is not necessarily the case (Wright 2019). Indeed, Priest \& Longcope (2017) found that the total amount of self-helicity in the final configuration depended upon the footpoint arrangement.

In the Priest \& Longcope (2017) model, an arcade of untwisted flux tubes (anchored in the same plane) was sheared parallel to a polarity inversion line, yielding running reconnection along the arcade (in a manner consistent with observed two-ribbon flare motions). This was termed "zipper reconnection." Threlfall et al. (2018b) numerically modeled the shearing and reconnection of a single pair of flux tubes (a "zippette"), finding that the model is indeed capable of forming a large twisted flux rope overlying a sheared arcade of field lines. In order to further explore the concept of helicity partitioning in the context of such results, Wright (2019) derived expressions that describe the role of footpoint positions on the amount and 
partitioning of magnetic twist in flux tubes that are anchored in the same plane and undergo reconnection.

The primary goal of this investigation is to further examine the "helicity equipartition conjecture" by testing whether the formulae of Wright (2019) can accurately predict the final helicity and twist of a magnetohydrodynamic (MHD) flux tube reconnection model, where the flux tubes are anchored in the same plane. To achieve this, we develop and implement a new practical method to determine the self-helicity and twist of a (simulated) magnetic flux tube. The structure of our paper is as follows: in Section 2 we outline some fundamental theoretical concepts of magnetic helicity. Details of our practical implementation, which relies upon some of these concepts, takes place in Section 3, where we outline our method to calculate flux tube self-helicity, subdividing each tube into small elements and analyzing the mutual helicity of each elemental pair. In Section 4 we describe the tests used to assess our method, comparing our recovered helicity values to an analytical model whose self-helicity is determined by a controllable number of turns. Following these tests, Section 5 outlines our application of the method to an MHD reconnection experiment, where two tubes are sheared and allowed to reconnect, with our findings described in Section 5.3. Finally, a discussion of our results is presented in Section 6, before conclusions are presented in Section 7.

\section{Theory}

We exploit properties of relative helicity (Berger \& Field 1984) to study a system of magnetic flux tubes anchored in the same plane as

$$
H=\sum_{i} H_{i}+\sum_{\substack{i, j \\ i \neq j}} H_{i j} .
$$

Equation (1) breaks the total system helicity into the individual (or self-)helicity of each flux tube combined with the mutual helicity of each flux tube relative to the others.

If the system contains a single flux tube with a crosssectional flux $\Phi_{i}$, then the self-helicity of that flux tube can be calculated as

$$
H_{i}=L \Phi_{i}^{2}=(T+\mathcal{W}) \Phi_{i}^{2}, \quad \Phi=\int_{s} \boldsymbol{B} \cdot \boldsymbol{d} \boldsymbol{s} .
$$

Here $L$ is the mean net winding number of field lines contained within the tube, $T$ is the mean twist number of the field lines about the tube axis, and $\mathcal{W}$ is the writhe number of the tube axis (Equations (25) and (26) of Berger \& Field 1984; Berger $\&$ Prior 2006).

For the case of a flux tube with no writhe, $T$ has a simple interpretation: for a uniformly twisted tube, it corresponds to the number of turns field lines make about the tube axis. If the tube has a more complicated internal structure, we can still use $T$ as a useful nondimensional variable for quantifying the average structre within the tube and also to define their mean rotation angle $\tilde{\phi}=2 \pi T$. We shall use $T$ (in the sense of the equivalent twist for a writheless flux tube) later to provide a dimensionless measure of the self-helicity of a flux tube.

The mutual helicity can be determined by the relative positions of appropriate footpoints (Berger 1986). For tubes that start and end in the same plane, such as those considered in this work (with examples in Figure 1), angles made by the footpoints in the plane also depend on whether the tubes are crossed or uncrossed, leading to mutual helicity contributions of the form

$$
\begin{aligned}
& H_{i j}=H_{j i}=\frac{\rho_{1}+\nu_{1}}{2 \pi} \Phi_{i} \Phi_{j}, \\
& H_{i j}=H_{j i}=\frac{\rho_{2}-\nu_{2}}{2 \pi} \Phi_{i} \Phi_{j},
\end{aligned}
$$

where Equation 3(a) represents a crossed configuration (Figure 1(a)) and Equation 3(b) represents an uncrossed configuration (Figure 1(b)).

Berger (1986) calculated these results by starting with two well-separated tubes, which have zero mutual helicity. The tubes are then brought together to the configurations in Figure 1. During this process the footpoints rotate about each other and causes a flux of helicity across the boundary. The integral of this flux allows the mutual helicity of the final configuration to be determined. Note that these results apply to a planar boundary.

\section{Helicity Calculation Method}

The magnetic field of a large-scale flux tube like that in Figure 2 can be broken down into a set of contiguous elemental flux tubes which, for clarity, we call "flux tubules." As was first shown by Berger \& Field (1984), the total self-helicity of the large tube may alternatively be calculated by evaluating the mutual helicities of a large number $(N)$ of its constituent flux tubules, each containing a fraction of the total flux $\left(\delta_{\phi} \approx \Phi / N\right)$; in such a case, the total self-helicity (referred to as "internal helicity" in Berger \& Field 1984) of the flux tubules become vanishingly small at large $N$, with a relative error $\sim 1 / N$. As illustrated by Figure 2, an effective twist can then be deduced by equating the total mutual helicity of all the flux tubules with the self-helicity of the composite large tube.

Our goal is to practically implement this approach to calculate the self-helicity and twist of given flux tubes and validate the technique on test tubes of known self-helicity. We then apply the self-helicity calculation to an MHD simulation of reconnecting flux tubes which allows us to answer definitively whether the self-helicity obeys the equipartition conjecture or the non-equipartition formulae of Wright (2019).

\subsection{Calculating the Mutual Helicity of a Single Tubule Pair}

Before applying the method to an entire flux tube, we will first demonstrate the procedure used in calculating the mutual helicity of a single tubule pair. The steps in this procedure are illustrated by Figure 3 .

First, we construct a Cartesian grid of positions within the positive footpoint of the chosen flux tube, centered on the location of the peak positive field strength at the base, $B_{z}^{\max }(z=0)$. A contour of magnetic field strength (shown as a black oval on the left side of Figure 3 ) is used to define the outside of the flux tube at the base of the domain. We set this contour value to be $5 \%$ of $B_{z}^{\max }$, in order to avoid complications arising from tracing field lines in near-zero field regions. ${ }^{1}$ We define grid points beyond this contour as being "outside" the flux tube; such points are discarded. The grid

\footnotetext{
1 In flux tube models used in this investigation (including the model used to test the method), the field outside the tube rapidly falls to zero. Field lines traced at/near the tube edge (using an adaptive step size) can overstep into such weak/zero field regions, leading to inaccurate field-line solutions, which must be ultimately discarded from the helicity calculation.
} 


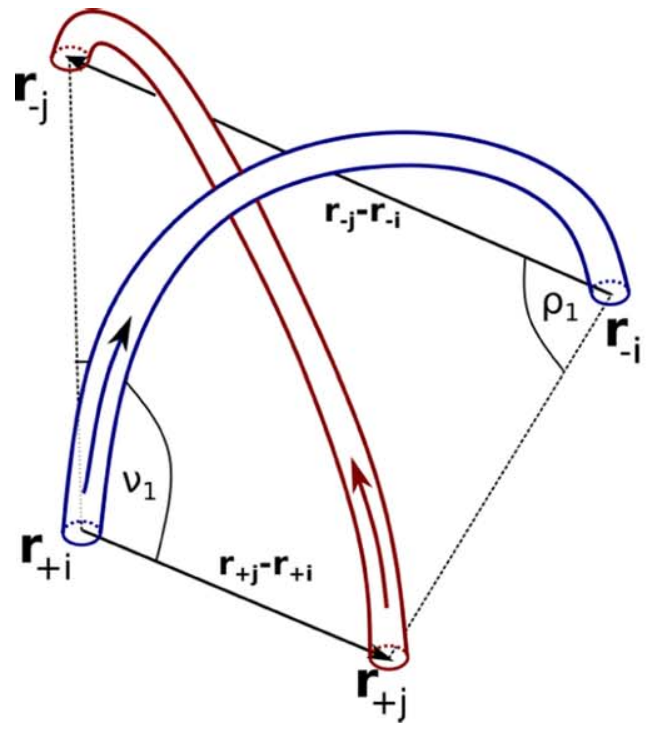

(a) Crossed Tubes

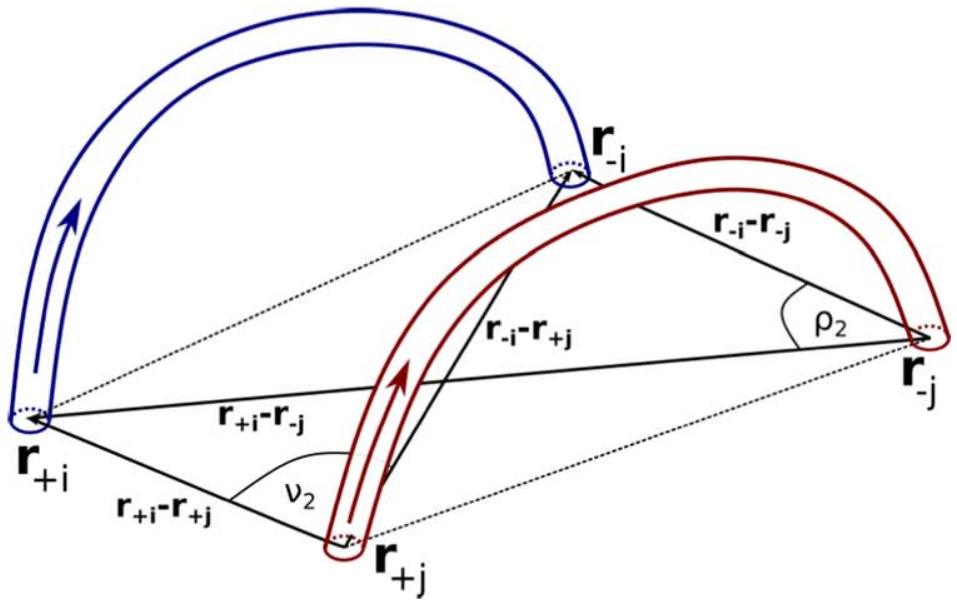

(b) Uncrossed Tubes

Figure 1. Illustration of how angles $\rho$ and $\nu$ are calculated, which depends on whether the tubes are (a) crossed and hence have mutual helicity calculated using Equation 3(a) or (b) uncrossed and hence have mutual helicity calculated using Equation 3(b); note that the color of the tube indicates whether we refer to the $i$ th (blue) or $j$ th (red) tube, while $\rho$ and $\nu$ are determined by specific vectors linking $i$ and $j$.

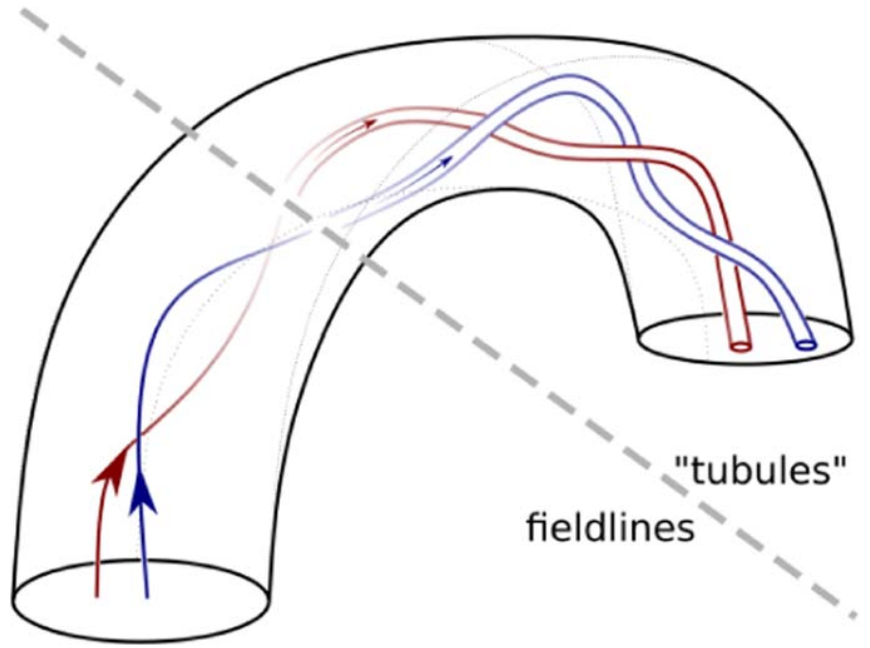

Figure 2. Total mutual helicity method of Berger \& Field (1984). The selfhelicity of an individual flux tube may instead be expressed as the sum of mutual helicities of many flux elements (small blue and red tubes) which together comprise the flux tube. To the left of the dashed gray diagonal line, the red and blue lines represent two field lines. These field lines form the axis of thin elemental flux tubes shown to the right of the dashed line.

spacing, $\delta_{x}, \delta_{y}$, controls the number of grid points $(N)$ in the flux tube.

At every grid point, we apply a numerical integration routine (which uses an adaptive step size to compare fourth- and fifthorder Runge-Kutta-Fehlberg solutions of the magnetic fieldline equations, used in, e.g., Threlfall et al. 2017, 2018a, 2018b) to trace a field line from the positive to negative footpoint of the flux tube. Field lines that also land outside a $5 \%$ contour of the negative field strength, $-B_{z}^{\max }(z=0)$, at the second footpoint are also omitted (though such instances are uncommon). The retained field lines effectively form the axes of our tubules, and comprise the entire flux tube volume. Each tubule associated with a field line has a cross-sectional area determined by the grid spacing $\delta_{x} \delta_{y}$,

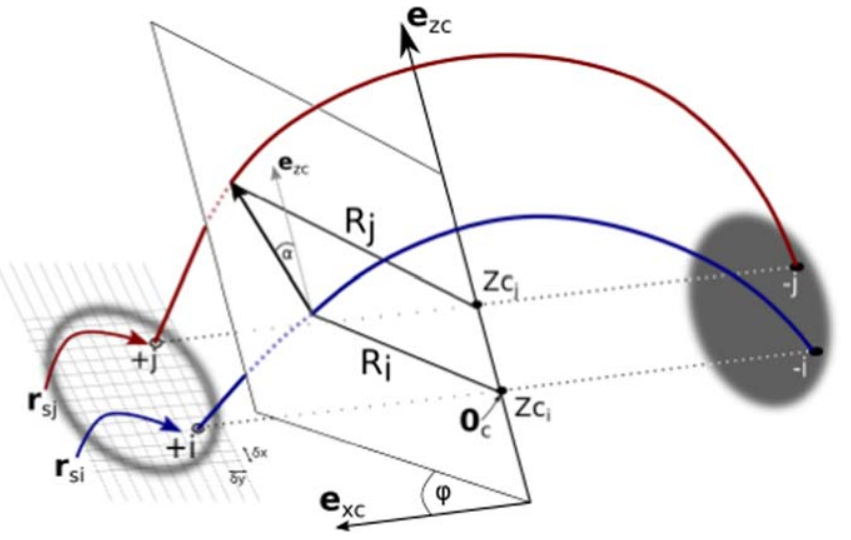

Figure 3. Method to calculate mutual tubule helicity. We calculate field-line trajectories (blue and red curves) from Cartesian grid points overlaid onto the positive footpoint of the flux tube, before calculating a new cylindrical coordinate system based on the relative initial and final positions of every fieldline pair, allowing us to calculate rotation angles $(\alpha)$ of one field line about another as a function of distance along the $i$ th field line, $s_{i}$.

while the flux is calculated as the perpendicular base flux at the starting point of each field line multiplied by this area $\Phi_{i} \approx B_{z}(z=0) \delta_{x} \delta_{y}$.

To account for extra windings of field lines between footpoints, we create a new cylindrical coordinate system aligned with our tubules; this new coordinate system is seen in Figure 3. A new $z$-axis $\left(\boldsymbol{e}_{z c}\right)$ is defined as the normal to a vertical plane containing the average start and end positions of each field-line pair; $\boldsymbol{e}_{z c}$ has no $z$ component (being located within the horizontal plane at the base of the domain). At an angle $\phi$ to this axis, we create a series of planes, with $\phi \in[0$, $\pi]$. $\phi=0$ corresponds to a plane extending over the positive tubule footpoints, while $\phi=\pi$ corresponds to a plane encompassing the negative tubule footpoints. The intercept of the $i$ th and $j$ th field lines with each $\phi$ plane is used to formulate a wrapping angle $\alpha_{i j}$ of the $j$ th field line around the $i$ th field line as a function of distance along the $i$ th field line. $\alpha_{i j}$ could, in 
principle, be interpreted directly as the twist of one field line around the other. However, our aim here is to simply count the number of complete turns of field line $j$ around field line $i$ : we subtract the initial from the final value $\left(\alpha_{i j}^{\text {final }}-\alpha_{i j}^{\text {initial }}\right)$ and evaluate the number of complete multiples of $2 n \pi$ (where $n$ can be positive or negative) that each field-line pair have turned about:

$$
n=\left\lfloor\frac{\alpha_{i j}^{\text {final }}-\alpha_{i j}^{\text {initial }}}{2 \pi}\right\rfloor
$$

As mentioned earlier, the mutual helicity of the tubes is determined from starting with unlinked tubes and moving their footpoints to the desired configuration. There is some ambiguity here as simply knowing the relative locations of the four footpoints does not account for the fact that the footpoint of one tube may have wound around the footpoint of the other tube an integer number of times without affecting the final footpoint locations. The effect of this is to add an integer number of links and an additional contribution to the mutual helicity of $n \Phi_{i} \Phi_{j}$ (Wright \& Berger 1990; Demoulin et al. 2006).

The final step is to combine the number of complete turns, $n$, with the footpoint angles ( $\rho$ and $\nu$ ) and the fluxes of both tubules to calculate the mutual tubule helicity. The definition of angles $\rho$ and $\nu$ changes depending on whether the tubes are crossed or uncrossed (as shown in Demoulin et al. 2006 and references therein). We modify the relevant formulae to calculate the mutual tubule helicity in response to the number of additional turns of tubule $j$ about tubule $i$ as follows:

$$
\begin{gathered}
H_{i j}=\operatorname{sgn}\left(\alpha_{i j}^{\mathrm{end}}-\alpha_{i j}^{\mathrm{start}}\right) \Phi_{i} \Phi_{j} \frac{\rho_{1}+\nu_{1}}{2 \pi}+n \Phi_{i} \Phi_{j}, \\
H_{i j}=\frac{\rho_{2}-\nu_{2}}{2 \pi} \Phi_{i} \Phi_{j}+n \Phi_{i} \Phi_{j},
\end{gathered}
$$

where the mutual tubule helicity formula for crossed tubules, Equation 5(a), now accounts for the sign of the change in winding of $j$ around $i$ (which is accounted for in the sign of the fluxes in Equation 5(b)).

Having now calculated the value of $H_{i j}$ for each tubule pair, all that remains is to sum the individual $H_{i j}$ values (provided $j \neq i$ ) to calculate the total helicity of the flux tube. Dividing this value by $\Phi^{2}$ for total flux $\Phi\left(=\sum_{i} \Phi_{i}\right)$ generates the average number of turns of twist performed by field lines within the flux tube, or the average twist $\tilde{\phi}$.

It should be noted that our approach can only be applied to suitably smooth or well-behaved magnetic fields. For example, if a field line is highly contorted and doubles back to cross a $\phi=$ const. plane in Figure 3, then our method is not suitable, and a more general and robust method to calculate $H_{i j}$ is needed, such as that described in Figure 8 of Demoulin et al. (2006). If the magnetic field is amenable to our method, it has the advantage of allowing for computational efficiency. For example, if we take the order of $m$ numerical steps along a tubule, the number of operations in our method scales as $m$, whereas the general linkage integral scales as $m^{2}$. Our approach is most likely to be applicable to relaxed fields, although we show it can also work for the time-dependent simulations in Section 5 .

\section{Testing the Tubule Approach}

Prior to any scientifically relevant application of this method, we must first examine whether the method accuracy conforms to the predicted value $(1 / N$, first shown by Berger \& Field 1984) and what factors influence the results. To test the method we created a simple analytical magnetic flux tube model, which includes a region of (controllable) magnetic twist.

In order to gauge the effectiveness of our practical implementation, we applied the method detailed in Section 3 to the test model for a variety of uniform twists spanning $-2<T<2$. The exact value of helicity is given in terms of $T$ in Equation (2). The percentage difference between the value of $T$ imposed in the model and the value recovered by summing the mutual tubule helicity, using 250 tubules, is within the error of $1 / N=1 / 250=0.4 \%$ for all values of $T$.

This technique relies upon field lines (tubules) forming smooth arcs, distributing twist over large spatial length scales. Increasing the number of turns in the test model creates large amounts of twist over relatively short spatial scales. Such field lines may cross a $\phi=$ constant plane more than once and requires a more general treatment than our method. We have limited our tests to a relatively restricted range of $T$ for this reason and aim to apply our method in practice to weakly twisted flux tubes or situations where the twist is broadly distributed. This restriction is offset by the computational savings of this approach.

\section{Application to 3D MHD Reconnection Model}

The final stage of this investigation is the application of our mutual tubule helicity method to an MHD reconnection experiment where two initially untwisted magnetic flux tubes reconnect and form two new twisted flux tubes.

Our configuration is a refinement of the zippette reconnection model described in detail by Threlfall et al. (2018b). In that model, a pair of untwisted magnetic flux tubes were sheared, in order to generate a current sheet and leading to magnetic reconnection. Threlfall et al. (2018b) found that this process was capable of creating a large highly twisted flux rope linking the farthest positive and negative sources in the system. A second flux tube, lying underneath the highly twisted tube, was anticipated but not resolved in their simulation. A visual summary of the experiment phases can be found in Figure 4. Subsequently, Wright (2019) estimated the helicity and twist of the new connections formed in Threlfall et al. (2018b), based upon the approximate footpoint locations of the end state of the experiment.

In this investigation, we will recap several of the pertinent details of a new experiment based on the work of Threlfall et al. (2018b). This new experiment crucially results in the creation of two new flux tubes, due to modifications of the shearing and reconnection experiment phases. While highlighting these modifications, we will attempt to avoid repetition of shared aspects of the experimental setup; the remaining details can be found in the previous publication.

\subsection{MHD Equations and Initial Configuration}

The magnetic field evolution is calculated by solving the MHD equations using the Lagrangian remap code (Lare3D) described in Arber et al. (2001). This solves the following 
a)

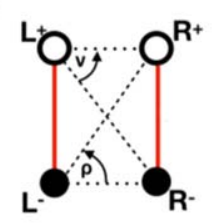

b)

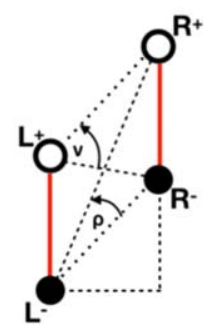

c)

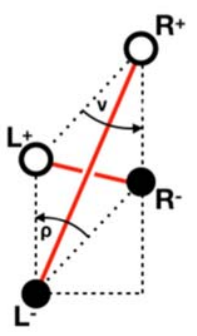

Figure 4. Adapted from Threlfall et al. (2018b). Cartoon illustration of the basic configuration (seen from above) of the zippette reconnection experiment, where two flux tubes reconnect and form new connections linking positive and negative sources. Solid red lines indicate flux tubes, while filled/empty circles indicate positive/negative source locations. The configuration in (a) is sheared to form that seen in (b), whereupon a current sheet (formed between the pair of flux tubes) reconnects, leading to (c) new connections between the flux sources (noting that the angles $\rho$ and $\nu$ which are required to calculate the mutual helicity of the configuration are not the same (b) before and (c) after reconnection takes place)

equations:

$$
\begin{gathered}
\frac{\partial \rho}{\partial t}+\nabla \cdot(\rho \boldsymbol{v})=0, \\
\rho \frac{\partial \boldsymbol{v}}{\partial t}+\rho \boldsymbol{v} \cdot \nabla \boldsymbol{v}=-\nabla p+\boldsymbol{j} \times \boldsymbol{B}, \\
\frac{\partial \boldsymbol{B}}{\partial t}=\nabla \times(\boldsymbol{v} \times \boldsymbol{B})-\nabla \times(\eta \nabla \times \boldsymbol{B}), \\
\frac{\partial p}{\partial t}+\boldsymbol{v} \cdot \nabla p=-\gamma p \nabla \cdot \boldsymbol{v}+\frac{j^{2}}{\sigma},
\end{gathered}
$$

for a plasma density $\rho$ and pressure $p$, at a velocity $\boldsymbol{v}$ and magnetic field $\boldsymbol{B}$, subject to a current density $\boldsymbol{j}=\nabla \times \boldsymbol{B} / \mu$ for magnetic permeability $\mu=4 \pi \times 10^{-7} \mathrm{H} \mathrm{m}^{-1}$, electrical conductivity $\sigma$, and magnetic diffusivity $\eta=1 /(\mu \sigma)$ (with the ratio of specific heats $\gamma=5 / 3$ ) and ensuring divergence-free magnetic field solutions $(\nabla \cdot \boldsymbol{B}=0)$. The dimensionless variables solved by Lare $3 \mathrm{D}$ are calculated assuming a magnetic field strength $B_{0}$, length $L$, and mass density $\rho_{0}$. For ease of comparison with Threlfall et al. (2018b), we will retain their normalizing choices, hence $B_{0}=10 \mathrm{G}, L=10^{7} \mathrm{~m}$, and $\rho_{0}=1.67 \times 10^{12} \mathrm{~kg} \mathrm{~m}^{-3}$, leading to a typical Alfvén speed of $V_{\mathrm{A}}=690 \mathrm{~km} \mathrm{~s}^{-1}$ and typical time $t=14.5 \mathrm{~s}$. The reference current density is $j_{0}=B_{0} /(\mu L)=8 \times 10^{-5} \mathrm{~A} \mathrm{~m}^{-2}$ and the reference magnetic diffusivity is $\eta_{0}=6.9 \times 10^{12} \mathrm{~m}^{2} \mathrm{~s}^{-1}$. Henceforth we will quote lengths $(L)$ in normalized units and times $(t)$ in Alfvén times, $\tau_{\mathrm{A}}=L / V_{\mathrm{A}}$ (based on the typical Alfvén speed). We also retain the same computational domain as that used in Threlfall et al. (2018b), $-8 \leqslant \bar{x} \leqslant 8$, $-8 \leqslant \bar{y} \leqslant 8$, and $0 \leqslant \bar{z} \leqslant 20$ (where barred quantities represent dimensionless variables in the numerical domain).

Our initial condition comprises two untwisted flux tubes with no writhe and constant cross section aligned with the positive $y$-axis, which then relax to form an equilibrium. The initial 3D configuration can be seen in Figure 5. The flux tubes in this experiment are larger and spaced more widely apart than those used in Threlfall et al. (2018b). The reason for this is that, while forming an equilibrium, the flux tubes expand to fill the rest of the computational domain (which contains no other magnetic flux). This expansion caused an overlap of the two sources (shown in, e.g., Figure 2 of Threlfall et al. 2018b),

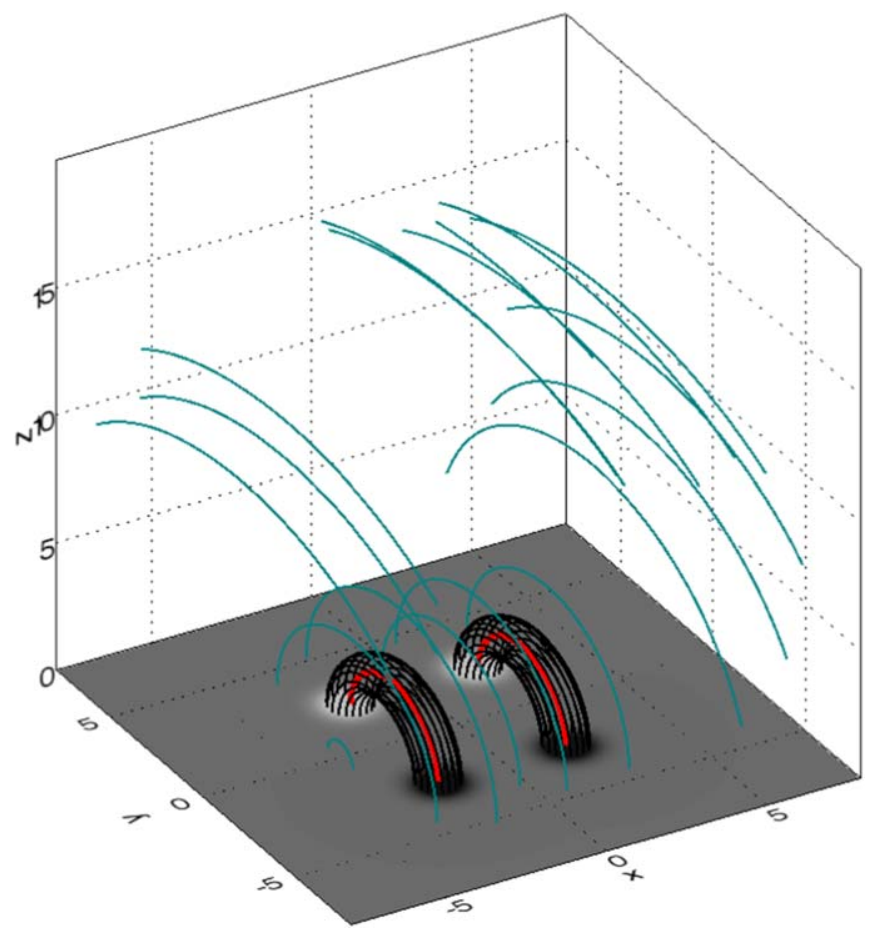

Figure 5. Initial 3D flux tube configuration, showing interpolated magnetic field lines which illustrate the flux tubes (black), the axis of each (red), and the ambient field (light blue).

requiring the omission of some flux in contours of base field strength in order to distinguish two distinct flux tube entities. In our new simulations, the wider tube separation allows us to define each flux tube using lower thresholds of vertical field at the base of the domain. Increasing the size of the flux tubes also allows us to trace more field lines within their volume compared to the Threlfall et al. (2018b) case.

In addition, our simulation uses a two-part magnetic diffusivity consisting of a background value, $\eta_{b}$, and an anomalous value, $\eta_{\mathrm{a}}$ (which is activated above a critical current threshold, $\left.j_{\text {crit }}\right)$. In Lare3D, this takes the following form:

$$
\eta=\left\{\begin{array}{cc}
\eta_{b} & |j|<j_{\text {crit }} \\
\eta_{a}+\eta_{b} & |j| \geqslant j_{\text {crit }}
\end{array}\right.
$$

Due to the excessive loss of magnetic flux from the sources due to background diffusivity in Threlfall et al. (2018b), we reduce the background resistivity $\eta_{b}$ by an order of magnitude from that investigation, hence $\eta_{b}=10^{-5}$ and $\eta_{a}=10^{-3} \cdot j_{\text {crit }}$ is chosen to reconnect a large laminar current sheet between the flux tubes.

\subsection{Shearing and Reconnection Phase}

Once in equilibrium, Threlfall et al. (2018b) sheared both sources using a velocity profile at the base, which smoothly ramped up (and down) in time using a tanh profile. The spatial variation of this profile was applied using a sinusoidal variation in $x$, which resulted in the cores of the sources moving apart as desired, but also generated additional v-shape "wings" at the edges of the sources (see e.g., Figure 3 of Threlfall et al. 2018b).

To limit the formation of similar wings on source profiles during our driving phase, we use a more complex form of the 


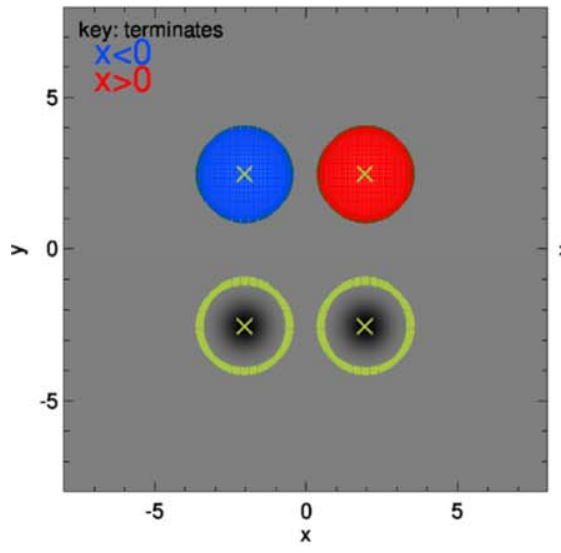

(a) $t=0$

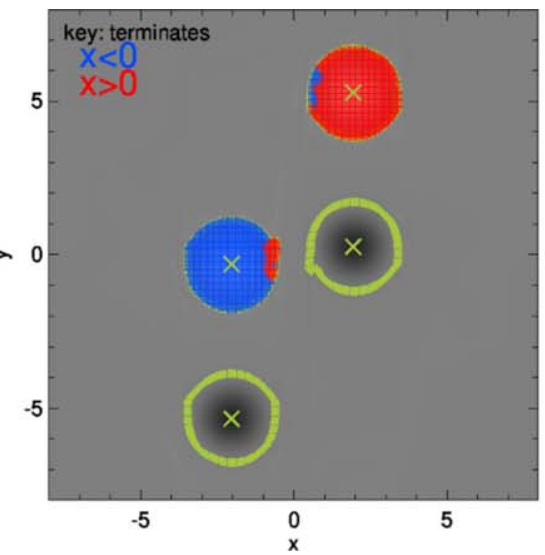

(b) $t=2200 \tau_{\mathrm{A}}$
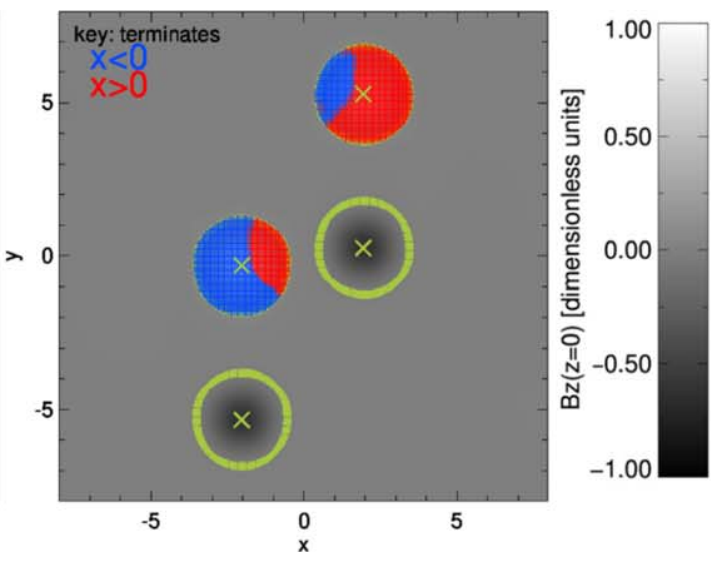

(c) $t=5000 \tau_{\mathrm{A}}$

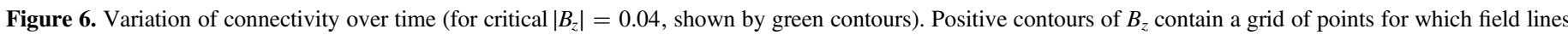

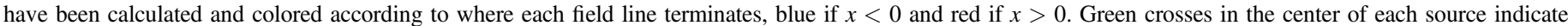
locations of $\min / \max B_{z}$.

driver, $v_{y}^{\text {base }}(t)$ :

$$
\begin{aligned}
& v_{y}^{\text {base }}(t)=\frac{A}{2}\left\{\tanh \left(\frac{t-2000}{2}\right)-\tanh \left(\frac{2200-t}{2}\right)\right\} \\
& \left\{\tanh \left(\frac{x-0.5}{0.1}\right)-\tanh \left(\frac{x-3.5}{0.1}\right)\right. \\
& \left.+\tanh \left(\frac{x+0.5}{0.1}\right)-\tanh \left(\frac{x+3.5}{0.1}\right)\right\},
\end{aligned}
$$

where $A=0.007$. Equation (11) improves flux retention/ conservation in the cores of the profiles during the driving phase (compared to the previous experiment), while still smoothly varying the spatial extent of the driver. Flux profile shape examples at different times can be seen in Figure 6. It should also be noted that by the end of the shearing phase, the shape of the profiles at the base of the domain remains large and roughly circular, unlike those seen in Threlfall et al. (2018b).

The shearing phase of our experiment is performed in ideal MHD; however, in Figure 6(b), a small region of weak magnetic flux has changed connectivity during the shearing phase. This can be attributed to numerical dissipation; once the resistive terms are activated after the shearing phase, larger regions of stronger magnetic flux are able to reconnect, as indicated by the large patches of red and blue formed in the positive sources seen in Figure 6(c) at the end of the experiment.

The effects of these changes to the experimental setup can be seen in the amount and linkage of magnetic flux between the sources during and after the shearing phase. This is shown in Figure 7. The key features of this image are (i) minimal loss of total flux over time and (ii) a final state in which approximately one-quarter of the total flux in each source is ultimately reconnected.

These experimental improvements also lead to the identification of two distinct flux tubes in the final state of the experiment, shown in Figure 8. Unlike Threlfall et al. (2018b), contours of the non-axial field form in the central $x, z$, and $y, z$ planes of the simulation, allowing us to identify two reconnected flux tubes whose connectivity matches that predicted in Figure 4(c). With both new flux tubes now

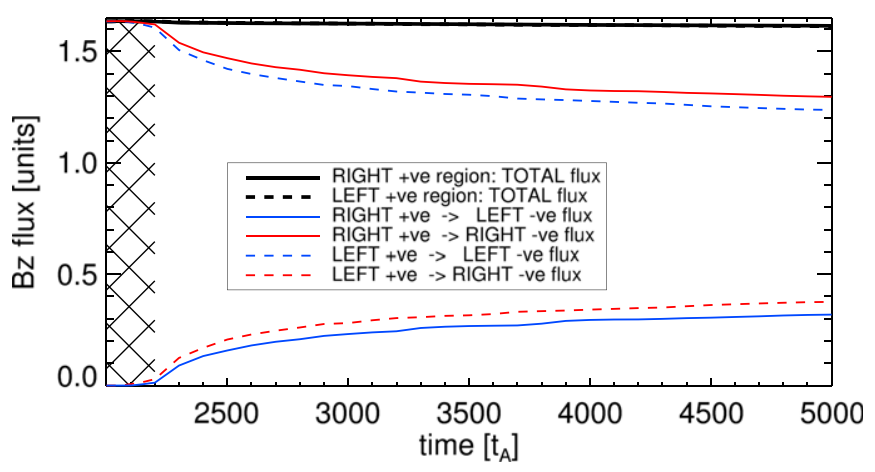

Figure 7. Time evolution of magnetic flux linkage between positive and negative sources in our MHD reconnection simulation both during and after the shearing phase. The key to the quantities is given in the legend.

containing a significant amount of the total flux, we are able to apply the mutual tubule helicity method to uncover the helicity in each new tube, and, hence, compare the helicity and twist with theoretical predictions (Wright 2019).

\subsection{Helicity and Twist Findings}

The theory underpinning our mutual tubule helicity method and our earlier tests show that the number of flux elements used are crucial in determining the approximate error of the helicity/ twist we calculate. We begin applying the method outlined in Section 3 by tracing field lines from the positive footpoint of the newly formed flux tubes, during each snapshot of the experiment, beginning at the start of the shearing phase. Figure 9(a) illustrates the number of field lines traced and their connectivity. With a method error that is inversely proportional to the number of field lines $N$ comprising each tube, Figure 9(a) illustrates that any estimates of helicity and twist in the reconnected flux tubes are associated with the largest errors at early times (when there are fewer reconnected field lines) compared to the end of the experiment $\left(t \sim 5000 \tau_{\mathrm{A}}\right)$. For example, at $t=2200 \tau_{\mathrm{A}}$, approximately 55 field lines thread the newly reconnected flux tubes and hence would be associated with a $1.8 \%$ error if used to calculate the mutual tubule helicity, compared to $N \approx 150$ at $t=5000 \tau_{\mathrm{A}}$, whereupon the error falls to $0.66 \%$. 


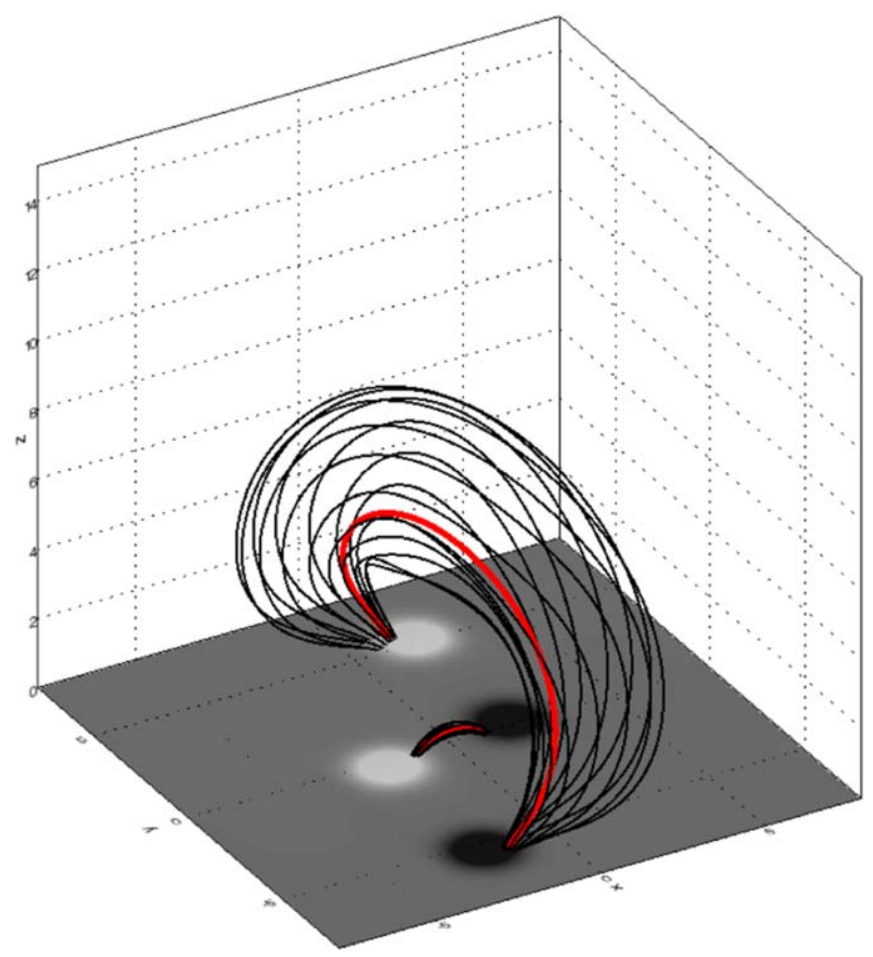

Figure 8. 3D magnetic field configuration, illustrating newly formed magnetic flux ropes at the end of the experiment. Magnetic field lines (seen in black) link a pair of regions of opposite magnetic polarity of $B_{z}$ at the base, with an axis indicated in red.

With this in mind, we applied our mutual tubule helicity technique to both flux tubes at various snapshots over the course of the simulations, yielding the signed helicity and twist evolution seen in the remaining panels of Figure 9.

Focusing on the recovered helicity values found in Figure 9(b), the helicity of the large overlying flux rope (blue dashed curve) appears well matched to the value calculated using the footpoint angles following Wright (2019; blue solid curve), particularly at later stages of the experiment. The sign of the helicity is negative, which is indicative of left-handed or counterclockwise twisting of the field lines that form the flux rope, as observed in 3D images of the field-line rotation of the upper tube in Figure 8. Meanwhile, the formulae of Wright (2019) predict a negligible net helicity in the lower tube (solid red curve); the recovered mutual tubule helicity (dashed red curve) is nonzero, but remains small (particularly when compared to the helicity of the overlying flux rope).

Dividing the helicities in Figure 9(b) by the square of the relevant fluxes found in each flux tube yields the average number of turns made by field lines over the length of each tube (when deformed to have $\mathcal{W}=0$ ), seen in Figure 9(c). Once again, the formulae of Wright (2019) predict a specific number of turns based on the angles made by the footpoints of the reconnected tubes (seen as solid lines in Figure 9(c)). We see that over time, the average twist in the overlying tube (dashed blue) approaches the value given by Wright $(2019 ; \sim-0.55$ based upon the footpoint configuration at $t=5000 \tau_{\mathrm{A}}$ ). However, the value predicted for the underlying flux rope (solid red line) is almost exactly zero, while the recovered twist of the underlying flux rope appears to diverge away from this value with time, achieving -0.03 turns by $t=5000 \tau_{\mathrm{A}}$.

It is noteworthy that a comparison of the predicted (from Wright 2019) and simulation twist at the end of the simulation illustrates that the theory overestimates the number of turns by 0.03 for the upper tube, and underestimates the twist in the lower tube by a similar amount. This is probably not a coincidence and is most likely due to the fact that as the prereconnection fluxes approach to reconnect, there is a little deformation that introduces some twist and writhe. As described in Wright (2019), the writhe and twist associated with such deformations must also be accounted for. In fact, we can surmise that the effect of bringing the tubes together to reconnect is to add 0.03 of a turn to the lower tube and to remove the same amount from the upper tube. See Figures 5(a) and (b) of Wright (2019) for an example of this effect.

\section{Discussion}

We have described a new practical approach to calculate the twist of a magnetic flux tube using mutual tubule helicity. Tests seen in Section 4 show that the method accuracy agrees with the original theoretical predictions of Berger \& Field (1984), with errors $\propto 1 / N$, where $N$ is the number of tubules (or field lines) used to subdivide the flux tube.

When applied to an MHD experiment modeling an observationally motivated case of flux tube reconnection in Section 5, our method recovers values of helicity and twist that closely match those predicted by Wright (2019). The key to Wright's helicity calculation is to recognize the importance of the writhe of the reconnected fluxes and is quite general as writhe is a property of the tube axis, not the cross-sectional form of the flux. Wright provided illustrative examples of his results using flux sheets to aid visualization. More recently, illustrative examples using flux tubes have been provided by Priest \& Longcope (2020) for the case where the footpoints of two uncrossed tubes form a parallelogram prior to reconnection.

\subsection{Potential Error Contributions}

In both the test configuration (Section 4) and MHD reconnection experiment (Section 5), the agreement between the expected and recovered values is good but not exact. As intimated above, the discrepancy is most likely due to the tubes being deformed to come together and reconnect. However, there are also possible errors associated with our numerical model that we consider further below.

Our numerical field-line integration routine underpins our technique and is a significant potential source of errors. We opted to use an adaptive (rather than constant) step size when solving the equations for each field line. This choice was made in order to improve computational efficiency (in light of the number of field lines required in order to yield an adequately small value of $1 / N$ ). However, the range of possible step sizes is critical and must remain relatively small, particularly for field lines that arc back to land in the same plane. Rapid step-size increases may lead to the code overshooting the original field line at a given point, while potentially still achieving RK4/5 solutions that agree. Overshoot is particularly problematic at the edge of the flux tube, requiring our tests to omit a thin shell of weak field at the flux tube edge.

Omission of this thin shell means that we have only analyzed the helicity of the majority of the central volume of the flux tube. The thin flux shell may only make minimal contribution to the total helicity, but in cases where multiple flux tubes reconnect (as in Section 5), this weak outer shell of flux will 


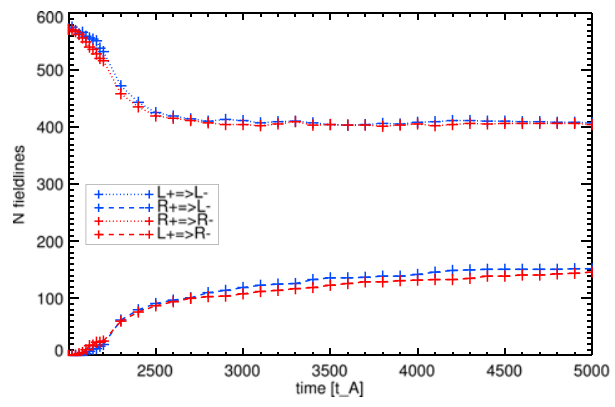

(a)Connectivity

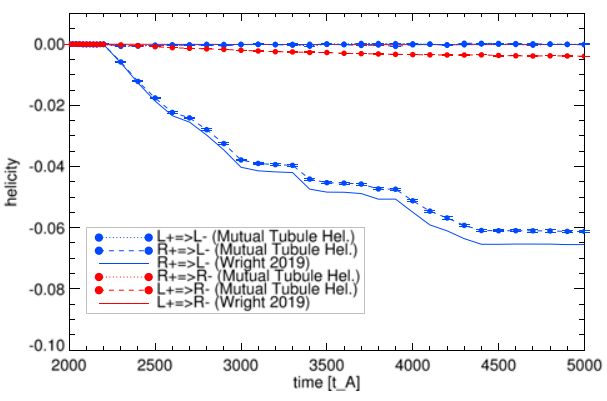

(b) Signed Helicity

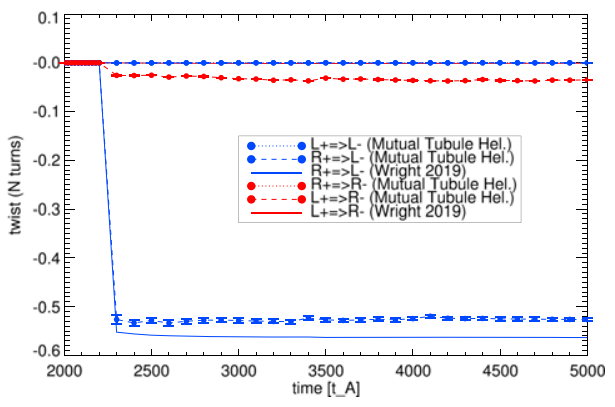

(c) Average Twist

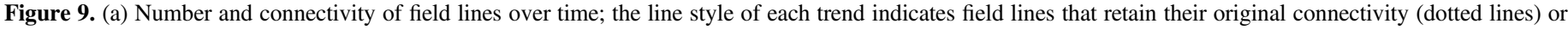

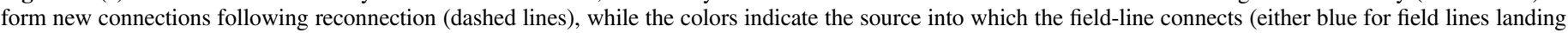

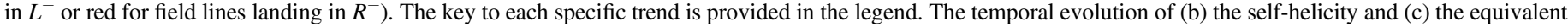

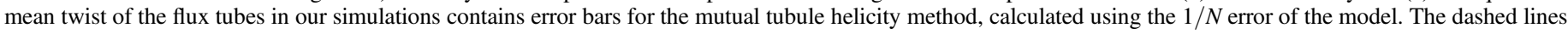

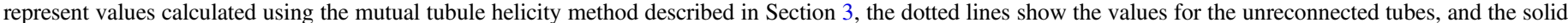

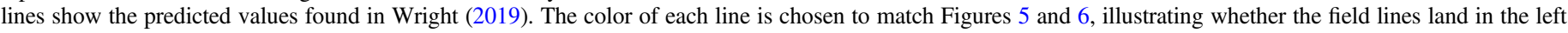
(blue) or right (red) negative sources (for key, see legend).

initially be the most dynamic, being the first part of the tubes to undergo reconnection; omission of this shell may therefore lose information about the helicity/twist at the earliest stages of reconnection. A potential remedy to this problem might be to solve field-line equations using a different geometry. Cylindrical field-line equation solutions (rather than our Cartesian approach, for example) could yield more accurate and faster results for field lines that are anticipated to smoothly form rounded arcs. Such arcs are more difficult to describe using Cartesian coordinates and hence require a much more careful choice of step size to balance accuracy with computational efficiency.

Finally, we must also take care to consider only field lines that morphologically adhere to our coordinate system. As mentioned in Section 4, field lines that contain many turns over a short spatial distance may become multivalued in the cylindrical coordinate system outlined in, e.g., Figure 3. In such cases, we must allow the twist to propagate along the structure or consider an alternative approach to calculate the self-helicity.

In the presence of nonzero $\eta$, helicity is not generally conserved and changes according to $d H / d t=-2 \int \eta \boldsymbol{j} \cdot \boldsymbol{B} d V$. This relation suggests that in the final snapshot $\Delta H / H \approx 7.5 \times 10^{-3}$, and so is conserved to better than $1 \%$ and indicates that the small discrepancy between predicted and simulation helicities is real and due to a small amount of twist and writhe introduced to the pre-reconnection tubes as they approach the reconnection site.

\subsection{Alternative Measures of Twist}

Liu et al. (2016) consider the structure and evolution of magnetic flux ropes in terms of twist. They define $\mathcal{T}_{w}$, the twist number of a given field line, and $\mathcal{T}_{g}$, the twist number of a field line about the axis of the flux rope. These two measures become the same for field lines near the tube axis, but not elsewhere. As Liu et al. (2016) were interested in the relation between twist and flux rope instability, $\mathcal{T}_{g}$ is the traditional key parameter used in classifying the behavior. However, this requires the accurate identification of the flux rope axis, and this is by no means trivial in simulation fields. They argue that $\mathcal{T}_{w}$ and its spatial variation can be used as a good indicator of the flux rope axis and so is employed as a proxy for $\mathcal{T}_{g}$. $\left(\mathcal{T}_{w}\right.$ is easy to compute on any field line without knowledge of the flux rope structure and location of the rope axis.)

Guo et al. (2017) also study magnetic flux ropes in terms of twist, writhe, and helicity. They use the formulation of Berger \& Prior (2006) to evaluate the twist and then estimate the selfhelicity of the flux rope by multiplying the twist by the axial flux squared. They found this estimate agreed best with the volume integral of the relative helicity density for just the current-carrying part of the rope. This is probably because the twist density is $\mu_{0} j_{\|} / 4 \pi B$ (Equation (16) of Berger \& Prior 2006) and will generally be largest at the axis of a flux rope. They also ignored the contribution of writhe to the selfhelicity (which is relatively small), but will affect the value they infer based upon twist alone.

The mutual tubule summation we employ will give the exact value of the self-helicity of the large composite flux tube (in the limit of a large number of tubules). If the self-helicity is divided by the axial flux squared, we arrive at a useful dimensionless number $L$ that is the mean net winding number which is conserved by ideal plasma motion. The mean net winding number is related to the twist and writhe by $L=T+\mathcal{W}$, where $\mathcal{W}$ is the writhe of the tube axis (Berger \& Field 1984 and references therein). Hence, we can imagine deforming the tube ideally so that it has $\mathcal{W}=0 \longrightarrow T=L=H_{i} / \Phi_{i}^{2}$. This is the sense in which we are using $T$ in Figure 9(c).

\section{Conclusions and Future Work}

We have presented a numerical simulation for the reconnection of fluxes anchored to a single plane. Our results give a clear answer to the question of whether the twist in the reconnected fluxes can be described using helicity equipartition (Priest et al. 2016; Priest \& Longcope 2017) or the nonequipartition formulae of Wright (2019): the non-equipartition formulae provide an excellent guide to estimating the selfhelicity and twist of the reconnected fluxes in our simulations. Moreover, our simulation exhibits extreme non-equipartition of helicity, with one tube having virtually no twist.

Our work has shown that mutual tubule helicity provides an accurate, reliable, and practical way to calculate the selfhelicity (and hence the average twist) of magnetic flux tubes in experiments that involve flux tube formation or reconnection, even in situations where the apparent twist of magnetic structures may be difficult to otherwise obtain. We have 
described the theory behind the mutual tubule helicity approach and our implementation and testing of this method in detail. Tests show that, in cases where the twist of the magnetic field is spread over a large spatial extent of a given flux tube, the error in the method agrees with theoretical predictions and is proportional to $1 / N$, the number of tubules into which the tube is broken up. Applying this tool to high-resolution MHD simulations of magnetic flux tube formation and reconnection shows that this approach is capable of recovering self-helicity values that were predicted by pre- and post-reconnection footpoint configurations (Wright 2019) to a high degree of accuracy. If the magnetic field is sufficiently smooth, we have presented a computationally efficient method for evaluating the helicity. For more complex magnetic fields, other more general formulations are required (see, e.g., Demoulin et al. 2006), although these are more computationally intensive. There are many configurations outlined in theoretical descriptions of magnetic helicity during reconnection, many of them in the same plane (Wright 2019). It would be meritorious to study to what extent this method agrees with such predictions, in which MHD reconnection takes place in other, more general footpoint configurations, before expanding this type of investigation to study flux tube configurations based on observed natural magnetic structures (for example, like those seen in the active solar corona).

The authors gratefully acknowledge the financial support of STFC through the Consolidated grant, ST/N000609/1, to the University of St Andrews. This work used the DIRAC 1, UKMHD Consortium machine at the University of $\mathrm{St}$ Andrews, the DiRAC Data Centric system at Durham University, operated by the Institute for Computational Cosmology, and the DiRAC Data Analytic system at the University of Cambridge, operated by the University of Cambridge High Performance Computing Service. These systems are operated on behalf of the STFC DiRAC HPC Facility (www.dirac.ac.uk). The equipment was funded by BIS National E-infrastructure capital grants (ST/K00042X/1 and ST/K001590/1), STFC capital grants (ST/K00087X/1, ST/ $\mathrm{H} 008861 / 1$ and ST/H00887X/1), and DiRAC Operations grants (ST/K003267/1 and ST/K00333X/1). DiRAC is part of the National E-Infrastructure.

\section{ORCID iDs}

James Threlfall (iD https://orcid.org/0000-0001-6690-0923

Andrew N. Wright (i) https://orcid.org/0000-0002-9877-1457

Alan W. Hood (ib https://orcid.org/0000-0003-2620-2068

\section{References}

Arber, T. D., Longbottom, A. W., Gerrard, C. L., \& Milne, A. M. 2001, JCoPh, 171,151

Asai, A., Yokoyama, T., Shimojo, M., et al. 2004, ApJ, 611, 557

Aulanier, G., Janvier, M., \& Schmieder, B. 2012, A\&A, 543, A110

Berger, M., \& Field, G. 1984, JFM, 147, 133

Berger, M. A. 1986, GApFD, 34, 265

Berger, M. A. 1988, A\&A, 201, 355

Berger, M. A., \& Prior, C. 2006, JPhA, 39, 8321

Démoulin, P. 2008, AnGeo, 26, 3113

Démoulin, P., Mandrini, C. H., van Driel-Gesztelyi, L., et al. 2002, A\&A, 382,650

Demoulin, P., Pariat, E., \& Berger, M. A. 2006, SoPh, 233, 3

Guo, Y., Pariat, E., Valori, G., et al. 2017, ApJ, 840, 40

Hood, A. W., Browning, P. K., \& van der Linden, R. A. M. 2009, A\&A, 506, 913

Liu, R., Kliem, B., Titov, V. S., et al. 2016, ApJ, 818, 148

Mackay, D. H., Green, L. M., \& van Ballegooijen, A. 2011, ApJ, 729, 97

Mackay, D. H., \& van Ballegooijen, A. A. 2006, ApJ, 641, 577

Moffatt, H. K. 1969, JFM, 35, 117

Priest, E. R., \& Longcope, D. W. 2017, SoPh, 292, 25

Priest, E. R., \& Longcope, D. W. 2020, SoPh, 295, 48

Priest, E. R., Longcope, D. W., \& Janvier, M. 2016, SoPh, 291, 2017

Russell, A. J. B., Yeates, A. R., Hornig, G., \& Wilmot-Smith, A. L. 2015, PhPl, 22, 032106

Taylor, J. B. 1974, PhRvL, 33, 1139

Threlfall, J., Hood, A. W., \& Browning, P. K. 2018a, A\&A, 611, A40

Threlfall, J., Hood, A. W., \& Priest, E. R. 2018b, SoPh, 293, 98

Threlfall, J., Neukirch, T., \& Parnell, C. E. 2017, SoPh, 292, 45

Vourlidas, A. 2014, PPCF, 56, 064001

Warren, H. P., O’Brien, C. M., \& Sheeley, N. R. J. 2011, ApJ, 742, 92

Webb, D. F. 2000, JASTP, 62, 1415

Wright, A. N. 2019, ApJ, 878, 102

Wright, A. N., \& Berger, M. A. 1989, JGR, 94, 1295

Wright, A. N., \& Berger, M. A. 1990, JGR, 95, 8029

Yeates, A. R., \& Hornig, G. 2016, A\&A, 594, A98

Yeates, A. R., \& Mackay, D. H. 2009, ApJ, 699, 1024 